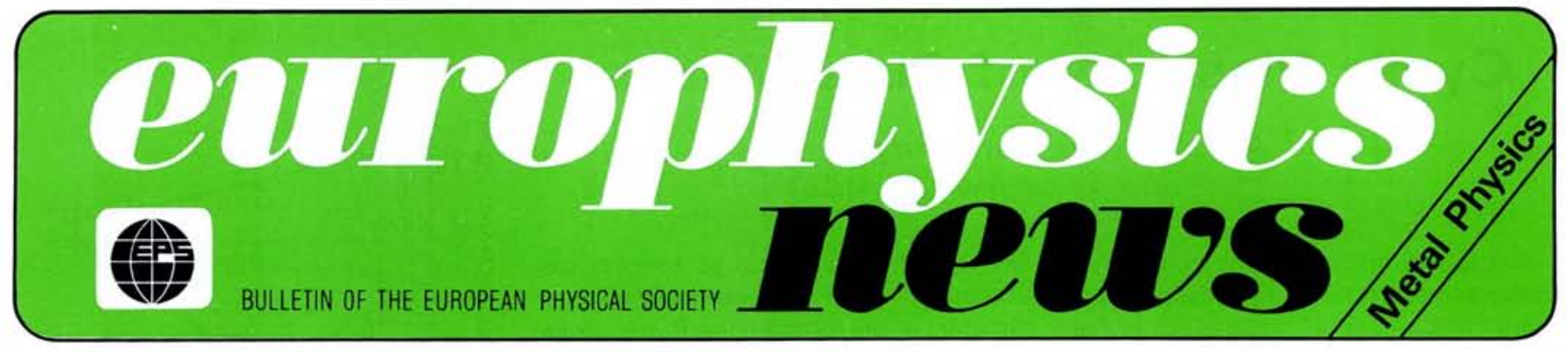

J.A.

Volume 14

Number 1

January 1983

\title{
Recent Advances in Physical Metallurgy
}

There must be few sections of the EPS with as broad a mandate as that of metal physics. Our members' interests stretch from the microscopic to the impressively macroscopic; we concern ourselves with both the nature of interatomic bonds and the performance of large engineering structures. It has been said that metallurgy is one of man's most ancient arts but one of his youngest sciences. The Bronze Age probably started with the chance observation of smelting effects in primitive hearths some five thousand years ago, but many of the phenomena now known to dictate the behaviour of a metallic component were observed and explained only during recent decades; it is, for example, intriguing to reflect on the fact that the neutron was discovered before that key to so much of our endeavour: the crystal dislocation. The evolution of our domain continues with undiminished vigour, as attested by this special edition devoted entirely to modern metal science. The following pages describe recent developments in a necessarily restricted, but we feel representative selection of our activities.

The design criteria for the metallurgist's products become increasingly stringent. In the face of such demands there is no room for trial and error. Today's metallurgist must understand his materials at the atomic level, know their ideal structure, and establish which imperfections are present, because the latter determine the microstructure and through this the macroscopic properties.

The 1983 Hewlett-Packard Europhysics Prize has been awarded to:

Professor Isaak F. Silvera

until recently at the University of Amsterdam for his outstanding contribution to our knowledge of:

Atomic and Solid Hydrogen.

Further details in the February issue.
Biologists and mineralogists can examine their thin specimens by transmitted light. But metals are opaque, and their study would seem, perforce, to be limited to observations of external surfaces.

This was indeed the situation for almost a hundred years, following Henry Sorby's pioneering development of the technique in which surface microstructure is made visible by the preferential etching effects of certain chemical reagents. This metallographic method, dating from 1864, reveals the grain size and shape, and also the state and distribution of precipitates. In many situations, this information is still sufficient to characterize a metal and predict its mechanical properties. It was Robert Heidenreich, in the late 1940s, who opened the door to the internal structure of metals, by exploiting their transparency to electrons of sufficiently high energy. And the application of transmission electron microscopy burgeoned after it was demonstrated that dislocations could be distinguished, and even seen to move. This advance was achieved simultaneously by Peter Hirsch, Robert Horne and Michael Whelan who, true to the tradition of the smithy, produced their thin specimens with hammer and anvil, and Walter Bollmann, whose electrochemical approach produced the greater control now regarded as standard practice. Erwin Müller took things a step further; his fieldion microscope actually enables one to "see" individual atoms, and thus detect even the smallest defects: vacancies, interstitials and impurity atoms. These pioneers, physicists all, essentially erased the boundaries between their discipline and metallurgy. Their efforts also showed how obvious things can appear in retrospect. Ulrich Dehlinger's "Verhakung" which we now call a dislocation, is instantly recognizable to anyone familiar with the construction of a Romanesque arch.

At the technological level, however, metallurgical problems seldom admit of simple predictions or easy solutions. It is not always easy to tell if a defect should be seen as hero or villain: dislocations can both promote strengthening and provoke cracking; vacancies mediate diffusion but cause swelling. And many different defects are on stage simultaneously; the plot is usually difficult to follow because too many characters are getting into the act. A case in point is the failure under cyclic stress known as fatique. It arises in a host of relatively prosaic situations, but is popularly associated with its dramatic impact on aviation. Understandably so; the prototype De Havilland 110 broke up in mid-air, above a crowd of a 100000 people at Farnborough in 1952, and the mysterious Comet disasters occurred just over a year later. Nevil Shute's No Highway presented the facts as they then appeared, even if speculations about melting atoms were a bit farfetched.

Our field, however, is not only concerned with trouble-shooting; in architecture, for instance, it makes an esthetic contribution by offering a variety of textures and colours. Copper roofs have long been in evidence, of course, and our cities are becoming metallurgical show-cases for anodized aluminium, stainless steel, bronze, and iron deliberately rusted. Metals will no doubt be displaced by other materials in specific applications. The plastic bicycle is already with us for example, but metals seem destined to play a continuing role in our society.

\section{R.M.J. Cotterill, \\ Chairman: Metal Physics Section, EPS Condensed Matter Division}

\section{CONTENTS}

\section{Recent Advances}

in Physical Metallurgy

Cyclic Plasticity of Pure Metals

Superplasticity of Metals

Metallurgy and Superconductivity

Metallic Glasses

The Surfaces of Metals

1
2
4
6
8
10

